\title{
Mortality after cancer among patients with diabetes mellitus: effect of diabetes duration and treatment
}

\author{
Kristina Ranc • Marit E. Jørgensen • Søren Friis • \\ Bendix Carstensen
}

Received: 23 October 2013 / Accepted: 20 January 2014 / Published online: 15 March 2014

(C) Springer-Verlag Berlin Heidelberg 2014

\begin{abstract}
Aims/hypothesis The prognostic role of different diabetes treatment types has not been studied in detail. We compared mortality rates among cancer patients with and without diabetes, accounting for diabetes treatment and diabetes duration. Methods This register-based study included all cancer patients diagnosed in Denmark during 1995-2009. The patients were classified into four groups according to diabetes status at the time of cancer diagnosis: no diabetes, diabetes without medication, diabetes with only oral hypoglycaemic agent (OHA) or diabetes with insulin treatment. Poisson models were used to examine the association between pre-existing diabetes in cancer patients and mortality relative to the nondiabetic cancer population.

Results Among 426,129 patients with incident cancer, we identified 42,205 patients with diabetes prior to cancer diagnosis. Overall, cancer patients with diabetes had higher mortality rates than non-diabetic cancer patients, highest among OHA- or insulin-treated patients. For all cancers combined and diabetes duration of 2 years at cancer diagnosis, insulintreated patients experienced the highest mortality rate ratios starting from 3.7 (95\% CI 2.7, 5.1) for men and $4.4(3.1,6.5)$ for women 1 year after cancer diagnosis, increasing to $5(3.5,7.0)$
\end{abstract}

Electronic supplementary material The online version of this article (doi:10.1007/s00125-014-3186-z) contains peer-reviewed but unedited supplementary material, which is available to authorised users.

K. Ranc $(\varangle) \cdot$ M. E. Jørgensen · B. Carstensen

Steno Diabetes Center, 2820 Gentofte, Denmark

e-mail: ksre@sund.ku.dk

K. Ranc $\cdot$ B. Carstensen

Department of Biostatistics, University of Copenhagen,

Copenhagen, Denmark

S. Friis

Danish Cancer Institute Research Center, Danish Cancer Society,

Copenhagen, Denmark for men and $6.5(4.2,9.3)$ for women 9 years after cancer diagnosis.

Conclusions/interpretation Our study provides strong evidence that cancer patients with pre-existing diabetes experience higher mortality than cancer patients without diabetes. The higher mortality seen among cancer patients treated with OHAs or insulin is in accordance with the existing evidence that more intensive diabetes treatment reflects a larger degree of comorbidity at the time of cancer diagnosis, and hence poorer survival.

Keywords Cancer · Diabetes duration · Insulin · Mortality · Oral hypoglycaemic agent $\cdot$ Register study $\cdot$ Type 2 diabetes

\begin{tabular}{|c|c|}
\hline \multicolumn{2}{|c|}{ Abbreviations } \\
\hline CRS & (Danish) Civil Registration System \\
\hline DCR & Danish Cancer Registry \\
\hline $\mathrm{DM}+\mathrm{Ins}$ & 'Diabetes and insulin' category in analysis \\
\hline DM NoMed & $\begin{array}{l}\text { 'Diabetes but no glucose-lowering medication } \\
\text { history' category in analysis }\end{array}$ \\
\hline $\mathrm{DM}+\mathrm{OHA}$ & 'Diabetes and OHA' category in analysis \\
\hline DPR & Danish Prescription Registry \\
\hline NDR & (Danish) National Diabetes Register \\
\hline NHISR & National Health Insurance Service Registry \\
\hline No DM & 'No diabetes' category in analysis \\
\hline NPR & National Patient Register \\
\hline OHA & Oral hypoglycaemic agent \\
\hline SMR & Standardised mortality ratio \\
\hline
\end{tabular}

\section{Introduction}

The association between type 2 diabetes, cancer and mortality is a complex topic receiving increasing recognition [1]. Strong evidence links type 2 diabetes and the risk of cancer 
occurrence [2-4]. This association differs for various cancer sites and in general is consistent among different studies.

A number of studies have reported that pre-existing diabetes influences the mortality of cancer patients, compared with cancer patients without diabetes $[5,6]$. However, a potential association between diabetes treatment and incident cancer and mortality has been addressed only in few studies $[4,6,7]$. In particular, little is known about the specific role of different diabetes treatment types, and findings differ among different studies [8-10], including the link between diabetes duration and mortality of cancer patients relative to the non-diabetic cancer population.

We aimed to explore differences in survival among cancer patients with diabetes prior to cancer diagnosis compared with cancer patients without diabetes. A specific aim was to examine the association between different types of glucoselowering therapies and survival after cancer diagnosis, as well as to examine how this association varies with duration of diabetes and by time since diagnosis of cancer.

\section{Methods}

Population and registries The study was conducted among all Danish patients diagnosed with cancer between 1 January 1995 and 31 December 2009. Using the unique personal identification numbers assigned to each resident in Denmark, all individuals from our study population were linked to (1) the Danish Civil Registration System (CRS); (2) the National Diabetes Register (NDR); and (3) the Danish Cancer Registry (DCR).

The CRS has assigned an unique personal identification number (CPR number) to all Danish residents since 1968. The CRS keeps continuous updated records for date of death or emigration of each individual, which enables unambiguous linkage between Danish registers [11].

The NDR was established in 2006 by linking the information from (1) the National Patient Register (NPR), which contains all hospital additions since 1994; (2) the National Health Insurance Service Registry (NHISR), containing the information about all service provisions provided by general and specialist practitioners to patients in Denmark, starting in 1973; and (3) the Danish prescription registry (DPR), which holds information on all prescriptions dispensed at Danish pharmacies since $1995[12,13]$. The inclusion date in the NDR was defined as the earliest of dates for hospital discharge with diabetes diagnosis (defined by ICD10: DE10-14, DH36.0, DO24, or ICD8 prior to 1999: 249, 250 www.who. int/classifications/icd/en/; http://www.wolfbane.com/icd/icd8. $\mathrm{htm}$ ) in the NPR; podiatry treatment for diabetes patients; the fifth glucose measurement date in 1 year; the last measurement date of two blood glucose measurements per year in 5 consecutive years in NHISR; the date of second oral glucose-lowering drug purchase within 6 months; or date of the second purchase of prescribed insulin in the DPR. Before 1995 the records on diabetes diagnosis were considered unreliable, hence the date of diabetes diagnosis could not be reliably established for cancer patients until 1995.

The DCR was established in 1943, and contains details of incident cancers in Denmark $[14,15]$. The DCR has been shown to have accurate and virtually compete registration of cancer cases. For the purpose of the study, we defined cancer diagnoses (excluding non-melanoma skin cancer) as the first primary cancer to avoid misclassification or potential effect of the treatment for any previous cancers.

Tabulation The date of cancer diagnosis was used as the inclusion date for all Danish cancer patients diagnosed from 1 January 1995 to 31 December 2009. In total, we identified 426,129 cancer patients who were followed until the date of death, emigration or the end of study follow-up (31 December 2009), whichever occurred first, providing a maximum follow-up of 15 years. The primary outcome of interest for this study was death, as recorded in the CRS.

The follow-up was classified by diabetes status at baseline in four groups: cancer patients without pre-existing diabetes (reference) (No DM; $n=383,924$ ), patients with diabetes but no history of glucose-lowering medication (DM NoMed; $n=15,453)$, patients exclusively treated with oral hypoglycaemic agents (OHAs) (DM + OHA; $n=17,708)$, or patients on insulin (DM + Ins; $n=9,044)$.

The analyses ware performed on all cancers combined, as well as separately for the 25 most common cancer sites. Table 1 presents person-years and the number of deaths of cancer patients during the follow-up in each of the four patient groups, subdivided by sex, for the whole population and for each of the 25 cancer sites.

All study participants were classified by diabetes status at baseline, sex, date of birth, and age and calendar time at follow-up defined in 1 year Lexis classes [16, 17]. For diabetic patients diagnosed after 1995, we have included diabetes duration (time from diabetes diagnosis until cancer diagnosis).

Statistical analysis The Cox proportional hazards model and the multiplicative Poisson model were used to examine the association between pre-existing diabetes in cancer patients and mortality, relative to non-diabetic cancer patients. The analysis was performed for cancer overall, separately for each sex and cancer site. The models were controlled for age, calendar time and diabetes status, all at the time of cancer diagnosis.

First, Cox models were fitted using time since cancer diagnosis as time-scale using only a fixed HR for each diabetes group compared with No DM.

To extend this idea, we modelled the rate ratios as a function of diabetes duration at cancer diagnosis, and as a function of time since cancer diagnosis relative to non-diabetic patients using Poisson models subdividing data into 1 month intervals 
Table 1 Number of person-years and deaths of cancer patients by diabetes status and sex for all cancers combined and for the 25 most common cancer sites

\begin{tabular}{|c|c|c|c|c|c|c|c|c|}
\hline \multirow[t]{2}{*}{ Cancer site } & \multicolumn{2}{|l|}{ No DM } & \multicolumn{2}{|c|}{ DM NoMed } & \multicolumn{2}{|c|}{$\mathrm{DM}+\mathrm{OHA}$} & \multicolumn{2}{|c|}{$\mathrm{DM}+\mathrm{Ins}$} \\
\hline & M & $\mathrm{F}$ & M & $\mathrm{F}$ & M & $\mathrm{F}$ & M & $\mathrm{F}$ \\
\hline Person-years & $218,666.3$ & $226,237.7$ & $8,173.5$ & $7,543.9$ & $11,072.7$ & $8,805.0$ & $4,866.1$ & $4,113.5$ \\
\hline All malignant neoplasms & 120,255 & 110,281 & 5,676 & 4,897 & 7,589 & 5,138 & 3,845 & 2,811 \\
\hline Oesophagus & 3,405 & 1,430 & 141 & 48 & 198 & 44 & 105 & 27 \\
\hline Stomach & 4,050 & 2,315 & 192 & 108 & 247 & 112 & 105 & 64 \\
\hline Ascending colon & 2,707 & 3,841 & 155 & 223 & 222 & 218 & 103 & 81 \\
\hline Transverse colon & 1,442 & 1,701 & 84 & 81 & 114 & 93 & 62 & 44 \\
\hline Descending and sigmoid colon & 5,022 & 4,547 & 252 & 200 & 339 & 215 & 138 & 106 \\
\hline Rectum & 5,930 & 4,230 & 262 & 158 & 362 & 205 & 158 & 67 \\
\hline Colorectal & 16,389 & 15,905 & 821 & 738 & 1,122 & 808 & 502 & 331 \\
\hline Liver & 1,796 & 1,123 & 198 & 73 & 345 & 81 & 211 & 56 \\
\hline Pancreas & 4,264 & 4,788 & 349 & 368 & 457 & 387 & 441 & 351 \\
\hline Lung, bronchus and pleura & 26,347 & 20,926 & 1,117 & 862 & 1,353 & 676 & 768 & 396 \\
\hline Melanoma of skin & 2,341 & 1,939 & 103 & 74 & 127 & 81 & 56 & 37 \\
\hline Breast & 172 & 19,142 & 6 & 692 & 7 & 890 & 5 & 430 \\
\hline Cervix uteri & - & 2,270 & - & 66 & - & 81 & - & 46 \\
\hline Corpus uteri & - & 3,320 & - & 196 & - & 256 & - & 120 \\
\hline Ovary, fallopian tube etc. & - & 5,711 & - & 194 & - & 185 & - & 97 \\
\hline Prostate & 18,139 & - & 816 & - & 1,129 & - & 394 & - \\
\hline Testis & 289 & - & 7 & - & 6 & - & 11 & - \\
\hline Kidney & 2,848 & 1,800 & 149 & 99 & 210 & 127 & 93 & 69 \\
\hline Urinary bladder & 9,185 & 3,362 & 397 & 130 & 615 & 162 & 241 & 79 \\
\hline Brain & 3,428 & 2,774 & 121 & 108 & 133 & 78 & 89 & 72 \\
\hline Thyroid & 227 & 361 & 5 & 17 & 13 & 14 & 6 & 14 \\
\hline Hodgkin's lymphoma & 263 & 170 & 12 & 10 & 14 & 11 & 8 & 5 \\
\hline Non-Hodgkin lymphoma & 3,218 & 2,718 & 148 & 114 & 167 & 132 & 78 & 69 \\
\hline Multiple myeloma & 1,910 & 1,473 & 88 & 70 & 92 & 59 & 46 & 33 \\
\hline Leukaemia & 3,683 & 2,767 & 162 & 124 & 192 & 120 & 110 & 57 \\
\hline Other & 19,589 & 17,573 & 912 & 882 & 1,247 & 911 & 617 & 491 \\
\hline
\end{tabular}

M, male; F, female

during the first 6 months, 3 month intervals during 7-12 months of follow-up, and thereafter 1 year intervals since cancer diagnosis. The models included age at cancer diagnosis, calendar time at cancer diagnosis and smooth terms for interaction between diabetes duration and status, and interaction between diabetes status at entry and time since cancer diagnosis, using natural cubic splines with five and four knots, respectively. The left boundary knot in diabetes duration term was chosen to be 0 in order to ensure that people with duration 0 and non-diabetic people were modelled with 0 contribution from the duration term. This model showed: (1) how diabetes status affected mortality as a function of time since cancer diagnosis; and (2) the mortality of diabetic relative to non-diabetic patients as a function of diabetes duration at cancer diagnosis. All data management and statistical analyses were performed in R (http://www.r-project.org/) [18].

\section{Results}

Median age of the population was 67 years among nondiabetic individuals (No DM), 73 years among both diabetic patients with no medication history or diabetic patients using OHAs (DM NoMed or DM + OHA) and 70 years among diabetic patients treated with insulin $(\mathrm{DM}+\mathrm{Ins})$. Median duration of diabetes at cancer diagnosis was $1.28,1.74$ and 1.65 years for the DM NoMed, DM + OHA and DM + Ins groups, respectively.

HRs for cancer mortality with $95 \%$ CIs and number of cases according to diabetes status and cancer site are presented in Fig. 1 for men and Fig. 2 for women (see also electronic supplementary material [ESM] Tables 1 and 2). For cancer overall, as well as for most specific cancer sites, mortality was higher among cancer patients with diabetes relative to non- 
Fig. 1 Estimated HRs of cancer mortality for male diabetic vs non-diabetic patients for each cancer site, with 95\% CIs. Bars on the right show the relative distribution of DM groups for each cancer site next to the number of deaths in each group. Desc., descending; Hodg., Hodgkin; lymp., lymphoma; mal., malignant; neop., neoplasms., sig., sigmoidal



diabetic patients. In all three diabetic groups the HRs followed a similar pattern for the specific cancer sites. For all cancer sites, there was a general tendency of increasing HRs according to diabetes treatment, i.e. patients using OHAs and insulin experienced higher mortality rates.

Statistically significant increases in mortality were observed among all three groups of diabetic patients for all cancers combined, and for colorectal, breast, ovary, cervix and corpus uteri, and bladder cancer. In addition, patients treated with insulin had a significant mortality increase for all previously mentioned cancers, as well as for rectum, lung and prostate cancer. For cancer overall, HRs were 1.10, 1.23 and 1.49 for men and 1.11, 1.22 and 1.46 for women in the DM NoMed, DM + OHA and DM + Ins groups, respectively. For colorectal cancer, the HRs were 1.10 for both male and female patients in the DM NoMed group, 1.17 and 1.22, for
Fig. 2 Estimated HRs of cancer mortality for female diabetic vs non-diabetic patients for each cancer site, with 95\% CIs. Bars on the right show the relative distribution of DM groups for each cancer site next to the number of deaths in each group. Desc., descending; Hodg., Hodgkin; lymp., lymphoma; mal., malignant; neop., neoplasms., sig., sigmoidal




male and female patients respectively in the DM + OHA group, and 1.44 and 1.29 , respectively, for the DM + Ins group. The corresponding HRs for breast cancer were 1.18, 1.42 and 1.68 , and for prostate cancer were $1.05,1.29$ and 1.46 , for the DM NoMed, DM + OHA and DM + Ins groups, respectively. Finally, HRs for lung cancer were 1.08 and 1.13 for OHA and insulin users, respectively.

Effect of diabetes duration and follow-up length We extended the simple proportional hazards model in order to explore the effect of diabetes status on mortality risk following cancer diagnosis as a function of diabetes duration at cancer diagnosis and as a function of time since cancer, relative to nondiabetic cancer patients.

Figure 3 shows the mortality rate ratios among patients in the three diabetic groups, with 2 years of diabetes duration at cancer diagnosis, relative to non-diabetic cancer patients, as function of time since cancer diagnosis in all diabetic groups for cancer overall. The rate ratios for cancer patients with preexisting diabetes were higher relative to non-diabetic patients and all three diabetic groups followed the same pattern. The mortality rate ratio curves revealed a small decline during the first year after the cancer diagnosis. However, after the first year there was a general increase in mortality rate ratios with increasing length of follow-up. Further, there was a tendency towards higher rates according to diabetes treatment, i.e. insulin-treated patients had the highest rates starting from 3.7 for men and 4.4 for women 1 year after the cancer diagnosis, up to around 5 for men and 6.5 for women after 9 years. Diabetic patients using OHAs or no glucose-lowering medications had lower rate ratios of around 1.10 for both sexes to about 1.5 for men and 2 for women during the same period. The site-specific plots are presented in ESM Fig. 1.

For the general Danish population (not subdivided by cancer status), we found that the standard mortality ratio (SMR) for diabetic vs non-diabetic patients was strongly dependent on age and duration of diabetes (See Fig. 5 in Carstensen et al [12]). Patients with 2 years of diabetes duration had a SMR ranging from 3 at age 50 years to 1.8 at age 70 years and 1.1 at age 80 years, with no difference between men and women.

Diabetes duration at cancer diagnosis did not influence the mortality estimates. There was a minor indication of a potential effect for patients with short diabetes duration at cancer diagnosis; however, overall there was no significant effect of diabetes duration (Fig. 4).

As inclusion dates of diabetic cases before 1995 are not reliable, we analysed them as a special subgroup of diabetes patients disregarding specific duration of diabetes. This specific group of patients includes a combination of the three defined treatment subtypes and could therefore not be directly compared with specific diabetic groups with specific diabetes duration at cancer diagnosis. The group of insulin-treated patients diagnosed before 1995 was quite small, exhibiting rate ratios of 1.08 for men and 1.74 for women. The corresponding rate ratios were 1.42 and 1.54 for non-treated and 1.01 and 1.38 for OHA-treated diabetes patients.

Absolute survival To further address the difference among cancer patients with or without pre-existing diabetes we also examined differences in absolute risks in order to estimate the expected survival of different DM groups of cancer patients.
Fig. 3 Mortality rate ratios for three groups of diabetic patients with 2 years of diabetes duration at cancer diagnosis compared with non-diabetic patients, as a function of time since cancer diagnosis, for all malignant neoplasms. Spline models with five parameters of diabetes duration at cancer diagnosis and four parameters of time since cancer diagnosis. Thin dashed lines indicate 95\% CIs. Blue, men; red, women




Fig. 4 Mortality rate ratios for three groups of diabetic patients relative to those with 2 years of diabetes duration at cancer diagnosis, as a function of diabetes duration, for all malignant neoplasms. Spline models with five parameters of diabetes duration at cancer diagnosis and four parameters of time since cancer diagnosis. Thin dashed lines indicate $95 \%$ CIs. Bars on the left are estimates for those diagnosed with DM before 1995. Blue, men; red, women

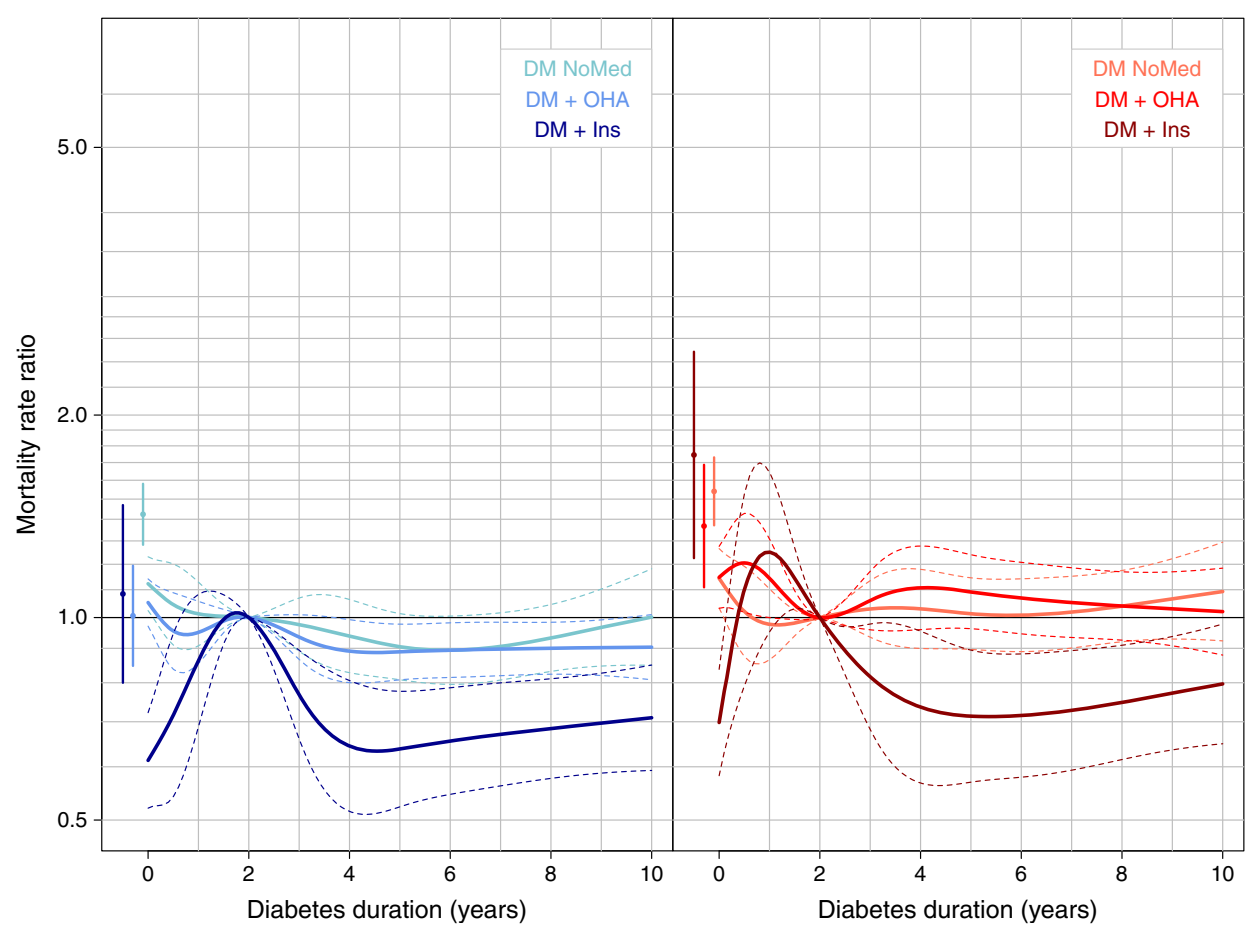

Figure 5 presents the cumulative survival probabilities of cancer patients aged 70 at cancer diagnosis without preexisting diabetes and three groups of diabetic patients with 2 years of diabetes duration at cancer diagnosis. In general, both sexes exhibited the same pattern, and insulin-treated cancer patients were associated with poorest expected survival. The survival probability decreased slowly with time since cancer diagnosis. Among men, we estimated a 20\% 2-year survival probability for insulin-treated patients. For OHAtreated patients and patients without history of diabetic therapy the 2 -year survival was about $60 \%$ and for cancer patients without diabetes almost $70 \%$. Survival was generally higher among women than among men, however, following the same pattern, that is around $30 \%$ for insulin-treated patients, $70 \%$ for OHA- and non-treated patients and $80 \%$ for non-diabetic patients. Site-specific plots are shown in ESM Fig. 2.
Fig. 5 Cumulative survival probabilities for non-diabetic and three groups of diabetic patients aged 70 with 2 years of diabetes duration at cancer diagnosis, as a function of time since cancer diagnosis for all malignant neoplasms. Spline models with five parameters of diabetes duration at cancer diagnosis and four parameters of time since cancer diagnosis. Thin dashed lines indicate $95 \%$ CI. Blue, men; red, women

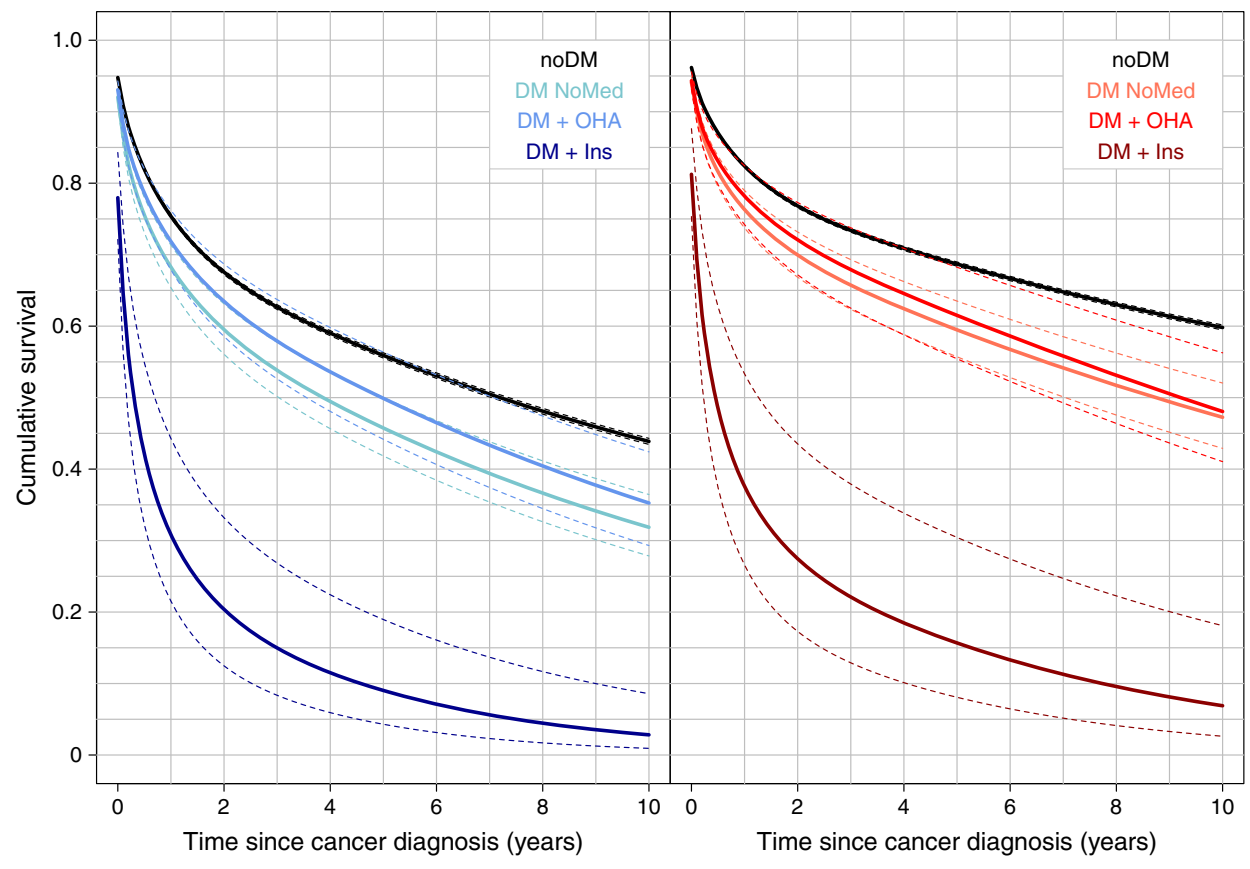




\section{Discussion}

Our study aimed to compare mortality among cancer patients with and without pre-existing diabetes, accounting for diabetes treatment type and diabetes duration. The results suggest that mortality of cancer patients with pre-existing diabetes is higher relative to non-diabetic patients for all cancers combined and for most individual cancer sites. In general, the highest mortality was found for OHA- or insulin-treated patients, suggesting that cancer patients with more intensive diabetes treatment have a larger degree of comorbidity at the time of cancer diagnosis, and hence poorer survival. Diabetes duration at cancer diagnosis did not influence the prognosis.

Further, we found a general increase in mortality over time since cancer diagnosis among diabetic patients relative to nondiabetic patients, which began 1 year after the cancer diagnosis. Mortality rates were higher with respect to more intensive treatment of diabetes, and highest for insulin-treated patients relative to non-diabetic patients.

Other studies investigating cancer survival related to diabetes status have also reported higher mortality related to treatment with insulin and sulfonylureas compared with metformin or no glucose-lowering treatment [5-10]. Several explanations for the observed association between pre-existing diabetes in cancer patients and increased all-cause mortality may be offered. The theoretical biological mechanism related to the increased mortality with insulin treatment relates to the hypothesis that hyperinsulinaemia brought about by exposure to insulin or by insulin resistance will accelerate the growth rate and thereby the progression of cancer. Furthermore, differences in cancer treatment between patients with and without diabetes may contribute to increased cancer-related mortality. Patients with diabetes often have other diabetes-related comorbid conditions, such as ischaemic heart disease, chronic kidney disease and neuropathy, that may influence clinical decisions [19].

In this context, van de Poll-Franse et al [20], in a Dutch study based on the Eindhoven Cancer Registry, found that diabetic patients with oesophageal, colon, breast or ovarian cancer were treated less aggressively than those without diabetes after controlling for age, cancer stage and sex. Further, patients with pre-existing diabetes may have poorer response to cancer treatment, including increased infection risk and intraoperative mortality. Finally, diabetic patients may on average present with more aggressive cancer at diagnosis than non-diabetic individuals, due either to masking of cancer symptoms because of symptoms related to diabetes or, theoretically, to an influence of diabetic therapy on the prediagnostic progression of the cancer. It is also possible that the excess mortality risk related to diabetes is independent of cancer and cancer treatment. Diabetes is a well-established risk factor for cardiovascular mortality in adults without cancer, and the microvascular and macrovascular damage associated with the disease is likely to induce disease and sometimes death regardless of cancer status. In our study, the excess mortality with diabetes was primarily seen for cancer types with long survival rates, such as breast, prostate and colorectal cancers, for which 'competing' cause of death is likely. This is in agreement with findings from other studies [5]. On the other hand, for cancers with short survival times, such as pancreas, liver and lung cancers, mortality was less influenced by diabetes or treatment status. Surprisingly, however, mortality after pancreas and liver cancer was largely unaffected by diabetes status even though diabetes and glucose-lowering drugs are strongly related to risk of these cancers. Such findings support the notion that the observed association between glucose-lowering treatment, particularly insulin, and cancer, is a result of reverse causality or confounding by indication due to progressive hyperglycaemia, hepatic insulin resistance and beta cell dysfunction already present in the initial cancer stages [21].

Strengths The study was conducted using nationwide data covering the entire Danish population, and thus there was no selection bias with regard to entry into the study population. Further, the available information on diabetes duration and treatment type (i.e. DM NoMed, DM + OHA or DM + Ins) facilitated computation of precise estimates of diabetes effect on survival after cancer diagnosis. Finally, the comprehensive Danish registration system ensured that all emigrations and deaths in the study population were properly accounted for.

Limitations We had no useful information on stage of cancer at diagnosis or specific cancer therapy. For most cancer sites, e.g. the prostate, the completeness and validity of cancer staging is low [22], and the available information in hospital registers on specific cancer therapy was also incomplete and only available from 2002 [23]. Hence, we were not able to explore whether differences in mortality were related to differences in severity of cancer disease at diagnosis or to differences in cancer treatment regimens. Information on medication use defined as redeemed prescriptions for OHAs or insulin did not include information on specific type or dosage.

Many factors influence the risk of both diabetes and cancer mortality, including comorbidities, obesity, insulin resistance, diet (including alcohol intake) and smoking history. The outcome of interest was death after cancer diagnosis; however, were not able to account for comorbidities or competing risks for death.

Conclusions Cancer patients with pre-existing diabetes experienced elevated mortality rates compared with the nondiabetic cancer population. Mortality was highest when diabetes and cancer diagnoses occurred within 1 year and among OHA- and insulin-treated patients. For all cancers combined, diabetic patients receiving no glucose-lowering medications 
presented long-term mortality rate ratios of 1.55 for men and 1.77 for women. The corresponding rate ratios were 1.52 and 2.01 among diabetic patients on OHA treatment, and 5.05 and 6.54 among insulin-treated patients, for men and women, respectively.

Diabetic patients using OHAs or insulin had higher mortality rates following stomach, colorectal, kidney, or bladder cancers, or melanoma of the skin or multiple myeloma. Diabetic patients with breast, uterus or ovarian cancer experienced higher mortality if treated with OHAs and highest mortality if treated with insulin. Men with prostate cancer and pre-existing diabetes also presented increased mortality if treated with OHAs or insulin.

In conclusion, our study provides strong support for the notion that pre-existing diabetes increases mortality among cancer patients, and that the excess mortality is larger among patients with diabetes treated with OHAs or, particularly, insulin. Several potential explanations for the impaired prognosis of cancer patients who also suffer from diabetes can be offered, including increased comorbidity, delayed cancer diagnosis due to masking of cancer symptoms, interaction with cancer therapy, and perhaps a direct effect of glucose-lowering regimens inducing more progressive cancer at diagnosis and/ or impaired prognosis. In any case, it is crucial that cancer patients with diabetes receive optimal diabetic treatment as well as any cancer-specific therapy; a therapeutic challenge typically demanding close collaboration between oncologists and endocrinologists.

\section{References}

1. Renehan AG, Yeh HC, Johnson JA, Wild SH, Gale EAM, Møller H (2012) Diabetes and cancer (2): evaluating the impact of diabetes on mortality in patients with cancer. Diabetologia 55:1619-1632

2. Carstensen B, Witte DR, Friis S (2012) Cancer occurrence in Danish diabetic patients: duration and insulin effect. Diabetologia 55:948958

3. Johnson JA, Carstensen B, Witte D, Bowker SL, Lipscombe L, Renehan AG (2012) Diabetes and cancer (1): evaluating the temporal relationship between type 2 diabetes and cancer incidence. Diabetologia 55:1607-1618

4. Wild SH (2011) Diabetes, treatments for diabetes and their effect on cancer incidence and mortality: attempts to disentangle the web of associations. Diabetologia 54:1589-1592
5. Barone BB, Yeh HC, Snyder CF et al (2008) Long-term all-cause mortality in cancer patients with preexisting diabetes mellitus: a systematic review and meta-analysis. JAMA 300:2754-2764

6. Currie CJ, Poole CD, Jenkins-Jones S, Gale EAM, Johnson JA, Morgan CL (2012) Mortality after incident cancer in people with and without type 2 diabetes. Diabetes Care 35:299-304

7. Bowker SL, Majumdar SR, Veugelers P, Johnson JA (2006) Increased cancer-related mortality for patients with type 2 diabetes who use sulfonylureas or insulin. Diabetes Care 29:254-258

8. Bowker SL, Yasui Y, Veugelers P, Johnson JA (2010) Glucoselowering agents and cancer mortality rates in type 2 diabetes: assessing effects of time-varying exposure. Diabetologia 53:16311637

9. Bo S, Ciccone G, Rosato R et al (2011) Cancer mortality reduction and metformin. A retrospective cohort study in type 2 diabetic patients. Diab Obes Metab 14:23-29

10. Baur DM, Klotsche J, Hamnvik OP et al (2010) Type 2 diabetes mellitus and medications for type 2 diabetes mellitus are associated with risk for and mortality from cancer in German primary care cohort. Metabolism 60:1363-1371

11. Pedersen CB (2011) The Danish Civil Registration System. Scan J Public Health 39(Suppl 7):22-25

12. Carstensen B, Kristensen JK, Ottosen P, Borch-Johnsen K (2008) The Danish National Diabetes Register: trends in incidence, prevalence and mortality. Diabetologia 51:2187-2196

13. Carstensen B, Christensen JK, Marcussen MM, Borch-Johnsen K (2011) The National Diabetes Register. Scan J Public Health 39(Suppl 7):58-61

14. Storm HH, Michelsen EV, Clemmensen IH, Pihl J (1997) The Danish Cancer Registry - history, content, quality and use. Dan Med Bull 44: 535-539

15. Gjerstorff ML (2011) The Danish Cancer Registry. Scan J Public Health 39(Suppl 7):42-45

16. Carstensen B (2007) Age-period-cohort models for the lexis diagram. Stat Med 26:3018-3045

17. Plummer M, Carstensen B (2011) Lexis: an R class for epidemiological studies with long-term follow-up. J Stat Softw 38(5):1-12

18. R Development Core Team (2012) R: a language and environment for statistical computing. R Foundation for Statistical Computing, Vienna

19. Richardson LC, Pollack LA (2005) Therapy insight: influence of type 2 diabetes on the development, treatment and outcome of cancer. Nat Clin Pract Oncol 2:48-53

20. van de Poll-Franse LV, Houterman S, Janssen-Heijnen ML, Dercksen MW, Coebergh JW, Haak HR (2007) Less aggressive treatment and worse overall survival in cancer patients with diabetes: a large population based analysis. Int J Cancer 120:1986-1992

21. Singh S, Singh PP, Singh AG, Murad H, McWilliams RR, Chari ST (2013) Anti-diabetic medications and risk of pancreatic cancer in patients with diabetes mellitus: a systematic review and metaanalysis. Am J Gastroenterol 108:510-519

22. Nguyen-Nielsen M, Froslev T, Friis S, Borre M, Harving N, Sogaard M (2012) Completeness of prostate cancer staging in the Danish Cancer Registry, 2004-2009. Clin Epidemiol 4:17-23

23. Lynge E, Sandegaard JL, Rebolj M (2011) The Danish National Patient Register. Scand J Public Health 39(Suppl 7):30-33 